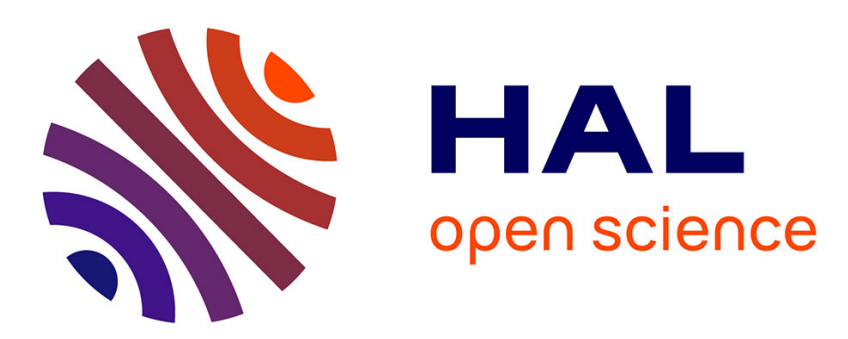

\title{
Fast determination of coarse-grained cell anisotropy and size in epithelial tissue images using Fourier transform
}

Mélina Durande, S. Tlili, T. Homan, B. Guirao, François Graner, Hélène

Delanoe-Ayari

\section{- To cite this version:}

Mélina Durande, S. Tlili, T. Homan, B. Guirao, François Graner, et al.. Fast determination of coarsegrained cell anisotropy and size in epithelial tissue images using Fourier transform. Physical Review E , 2019, 99 (6), pp.062401. 10.1103/PhysRevE.99.062401 . hal-02398787

\section{HAL Id: hal-02398787 \\ https://hal.science/hal-02398787}

Submitted on 4 Nov 2020

HAL is a multi-disciplinary open access archive for the deposit and dissemination of scientific research documents, whether they are published or not. The documents may come from teaching and research institutions in France or abroad, or from public or private research centers.
L'archive ouverte pluridisciplinaire HAL, est destinée au dépôt et à la diffusion de documents scientifiques de niveau recherche, publiés ou non, émanant des établissements d'enseignement et de recherche français ou étrangers, des laboratoires publics ou privés. 


\title{
Fast determination of coarse-grained cell anisotropy and size in epithelial tissue images using Fourier transform
}

\author{
M. Durande, ${ }^{1,2, *}$ S. Tlili,${ }^{1,3}$ T. Homan, ${ }^{2}$ B. Guirao,${ }^{4}$ F. Graner,,${ }^{1, \dagger}$ and H. Delanoë-Ayari ${ }^{2,+}$ \\ ${ }^{1}$ Laboratoire Matière et Systèmes Complexes, Université Denis Diderot - Paris 7, CNRS UMR 7057, 10 rue Alice Domon et \\ Léonie Duquet, F-75205 Paris Cedex 13, France \\ ${ }^{2}$ Univ. Lyon, Université Claude Bernard Lyon 1, CNRS UMR 5306, Institut Lumière Matière, Campus LyonTech - La Doua, Kastler building, \\ 10 rue Ada Byron, F-69622 Villeurbanne Cedex, France \\ ${ }^{3}$ Mechanobiology Institute, Department of Biological Sciences, National University of Singapore, 5A Engineering Drive, 1, 117411 Singapore \\ ${ }^{4}$ Polarity, Division and Morphogenesis Team, Institut Curie, CNRS UMR 3215, INSERM U934, 26 rue d'Ulm, 75248 Paris Cedex 05, France
}

(Received 16 November 2018; revised manuscript received 17 March 2019; published 4 June 2019)

\begin{abstract}
Mechanical strain and stress play a major role in biological processes such as wound healing or morphogenesis. To assess this role quantitatively, fixed or live images of tissues are acquired at a cellular precision in large fields of views. To exploit these data, large numbers of cells have to be analyzed to extract cell shape anisotropy and cell size. Most frequently, this is performed through detailed individual cell contour determination, using socalled segmentation computer programs, complemented if necessary by manual detection and error corrections. However, a coarse-grained and faster technique can be recommended in at least three situations: first, when detailed information on individual cell contours is not required; for instance, in studies which require only coarse-grained average information on cell anisotropy. Second, as an exploratory step to determine whether full segmentation can be potentially useful. Third, when segmentation is too difficult, for instance due to poor image quality or too large a cell number. We developed a user-friendly, Fourier-transform-based image analysis pipeline. It is fast (typically $10^{4}$ cells per minute with a current laptop computer) and suitable for time, space, or ensemble averages. We validate it on one set of artificial images and on two sets of fully segmented images, one from a Drosophila pupa and the other from a chicken embryo; the pipeline results are robust. Perspectives include in vitro tissues, nonbiological cellular patterns such as foams and $x y z$ stacks.
\end{abstract}

DOI: 10.1103/PhysRevE.99.062401

\section{INTRODUCTION}

During important physiological processes such as wound healing, morphogenesis, or metastasis, cells deform, migrate, exchange neighbors, divide, and die. A proper mechanical description of such a complex active system requires the characterization of cell size, cell shape, and changes thereof [1]. Fluorescent labeling of cell contours and progress in microscopy have led to the acquisition of large tissue images with high signal-to-noise ratio. Determination of individual cell contours have allowed the application of mechanical approaches based on quantitative data analysis of cell packing within epithelial tissues [2-4]; the development of quantitative modeling of tissue structure [5-7]; and even the linking of cell-level changes to morphogenetic movements [8-11]. While these studies were bidimensional, three-dimensional studies are becoming increasingly common [12-14].

These studies have been performed successfully by using detailed determination of cell contours, also called "cell segmentation," either manual, automatic, or a combination of both. Segmentation programs are available in an increasing number (see Refs. [11,12,15] and references therein). Figure 1

\footnotetext{
*melina.durande@univ-paris-diderot.fr

†francois.graner@univ-paris-diderot.fr

†helene.delanoe-ayari@univ-lyon1.fr
}

presents an example of such segmentation, in which the dorsal thorax of a fruit fly (Drosophila) is imaged at the pupal stage, i.e., during its metamorphosis from larva to adult. For each cell, the inertia matrix (see Appendix A) is computed and represented as an ellipse which fits the cell contour. It is completely defined by three parameters: its major and minor axis length $\left(L_{\mathrm{maj}}, L_{\mathrm{min}}\right)$ and the orientation $\theta$ of its major axis with respect to the $x$ axis. The inertia matrix can be averaged on any given region, yielding an average inertia matrix and thus an ellipse characterizing the average cell size and shape in this region. The cell area is characterized by $\pi L_{\mathrm{maj}} L_{\mathrm{min}}$.

The ratio $L_{\mathrm{maj}} / L_{\min }$ and the angle $\theta$ characterize the anisotropic part of the cell shape and are expressed by the anisotropic part of the inertia matrix. Interestingly, it is shown with image analysis by using force inference on detailed cell contours [10] that the anisotropic part of the inertia matrix correlates strongly with the anisotropic part of the stress at cell-cell junctions [Fig. 1(c)]. This last result is in agreement with a recent theoretical prediction [16] (under the assumption that cell-cell junctions and sizes are homogeneous in the tissue) and suggests that shape measurements could be used as a proxy to estimate stress (with exceptions recently documented in some extreme cases; see Ref. [17]). Moreover, the inertia matrix also correlates strongly with the texture tensor (see Appendix A, Fig. 8) that is used to statistically define the strain [18]. This reinforces the interest of cell 
(a)

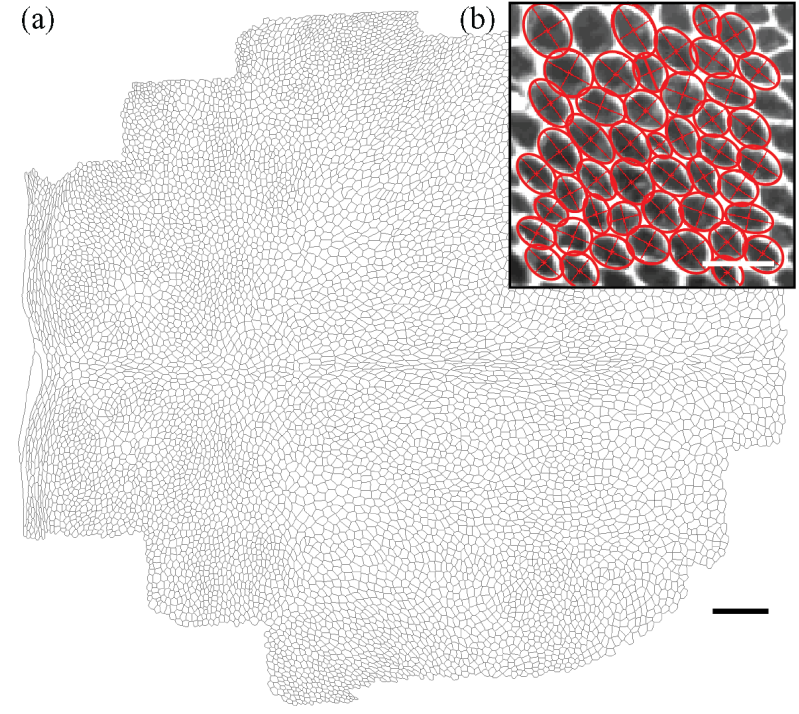

(c)

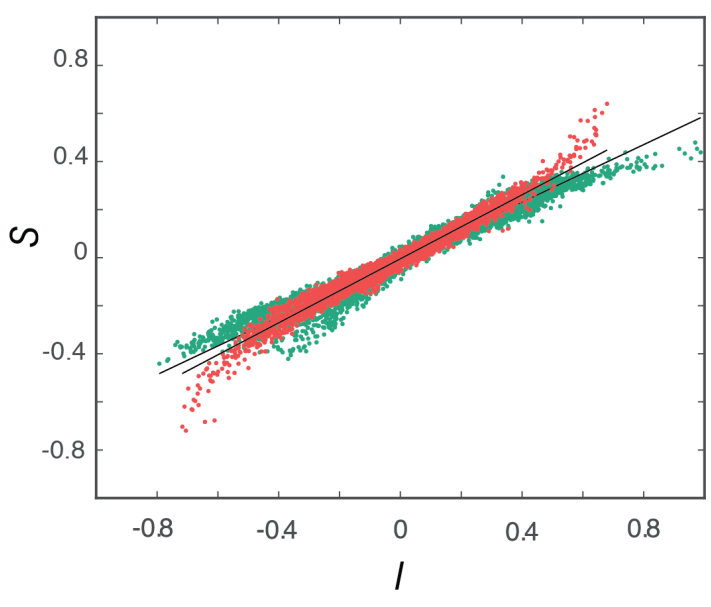

FIG. 1. Cell segmentation reveals a correlation between cell shape and elastic stress anisotropies. (a) Whole segmented image of the Drosophila dorsal thorax during its metamorphosis. Head is on the right of the image and abdomen on the left. Scale bar is $50 \mu \mathrm{m}$. (b) Subimage in original gray levels, scale bar is $10 \mu \mathrm{m}$. Cells are transfected to label the membrane with a fluorescent marker (green fluorescent protein, GFP), imaged by using an inverted confocal spinning disk microscope, and segmented by using a home-made software followed by automatic and manual rounds of correction [10]. In panel (b), the inertia matrix of each cell is superimposed as an ellipse. (c) Diagonal component (green) and off-diagonal component (red) of the cell-cell junction tension contribution to stress $S$, representing elastic stress anisotropy, versus the corresponding anisotropic (diagonal and off-diagonal) component of the inertia matrix $I$, representing cell shape anisotropy. Correlation coefficients are respectively 0.97 and 0.96 , slopes are respectively 0.6 and 0.67 . Tensors built with data from Ref. [10], here plotted after adimensionalization by the isotropic part of the respective tensor. To compute each of the 14112 points, tensors of individual cells are computed before being averaged in Eulerian grids of $40 \times 40 \mu \mathrm{m}^{2}$ with $50 \%$ overlap. Then a sliding average is performed on $2 \mathrm{~h}(24$ frames) time windows with a one hour overlap. Boxes at the pattern boundary which are filled at less than $30 \%$ by cells are excluded from the fit.

shape measurements as an approximative but fast and simple alternative to stress measurements. Since stress is defined as a coarse-grained quantity over a tissue region, average shape measurements should suffice without the need for detailed individual cell shape segmentation.

There are cases where a segmentation-free method of cell shape determination is potentially useful. For instance, a fast exploration of cell shape variation in time and space could help determine its role in a given biological question, before undertaking the detailed segmentation. Or, it could partially replace segmentation in cases where the image quality makes it difficult to segment with reasonable effort and sufficient precision: low or variable contrast, low signal-to-noise ratio, interrupted cell edges, large variability of cell sizes, variety of cell types or very contorted cell shapes. Even when the image can be segmented, the cell number can be much too large to enable segmentation within a reasonable amount of time.

Different techniques have been probed to quantify a pattern anisotropy without segmentation, such as the Hough transform [19], Radon transform [20], or Leray transform [21]. Fiber pattern anisotropy has been the subject of particular attention [22]. Fourier transforms (Fig. 2) (FTs) have already been used to determine the anisotropy of fibrous-like intracellular myosin distribution [23]. Fourier, Hilbert, and wavelet analysis are common in image analysis, with comparable performances when tested on common benchmarks $[24,25]$. One of the advantages of Fourier transform, beyond its simplicity, is that its amplitude (as opposed to its phase) is insensitive to small displacements of images; hence the
Fourier amplitude measured on successive images, images from different regions, or images from different experiments can be averaged [23].

Here, we implement a Fourier-transform-based pipeline which, in addition to all the above classical applications for pattern anisotropy quantifications, has specific advantages for the quantification of cellular patterns. It can determine the coarse-grained cell shape anisotropy in subregions of the whole image, resulting in cell shape anisotropy and orientation maps. Whenever the image quality is sufficient, it can determine the cell size, too. Note that it extracts the anisotropy and size of the averaged cell shape over a subregion (not the average of many individual cell anisotropies and sizes). Whenever it is known, or it can be reasonably assumed, that in the rest state the cell shape is isotropic, the cell shape anisotropy in the current state measures the cell strain deviator (see Appendix A); similarly, if the cell size in the rest state is known, the current cell size measures the cell strain trace. These two measurements are fundamental for determining the mechanical state of the tissue. We validate the pipeline with two already segmented images, in a Drosophila pupa and in a chicken embryo, and discuss its advantages.

\section{PIPELINE}

The Fourier transform (FT) of an ordered periodic pattern has peaks [Figs. 2(a)-2(c)]; the peak positions and intensities reveal the spatial periods and orientations present in the image. On the other hand, the FT of a disordered anisotropic 

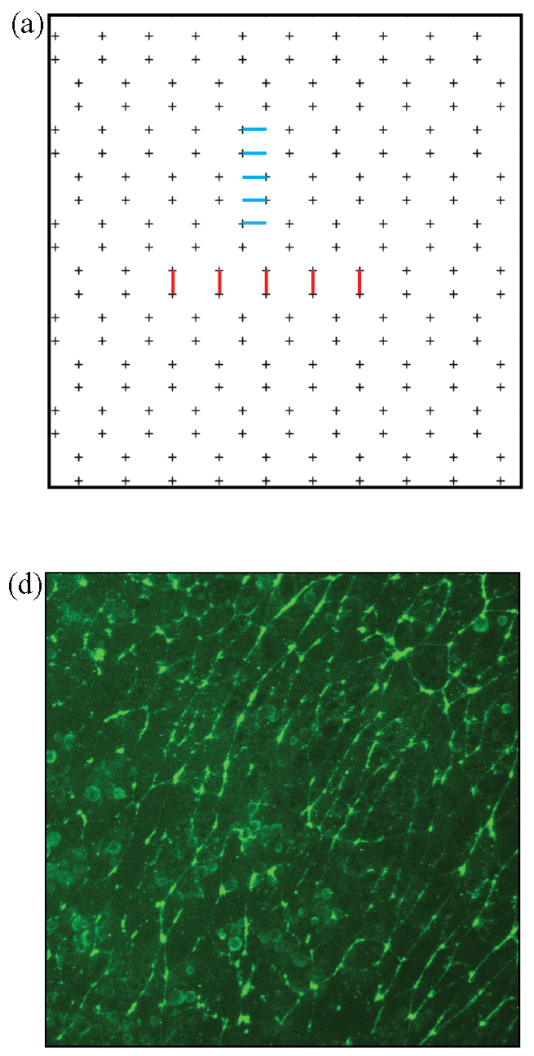

(b)

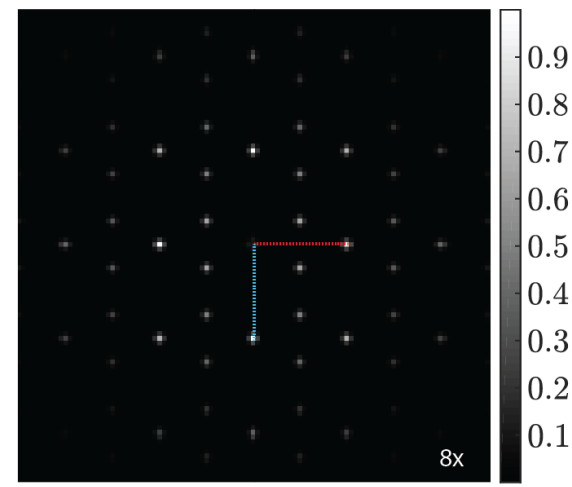

(e)

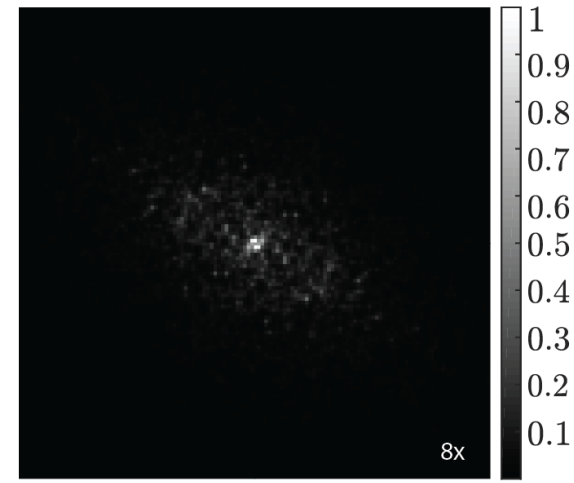

(c)

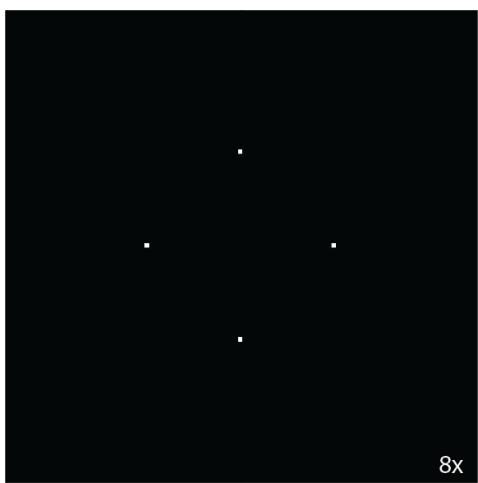

(f)

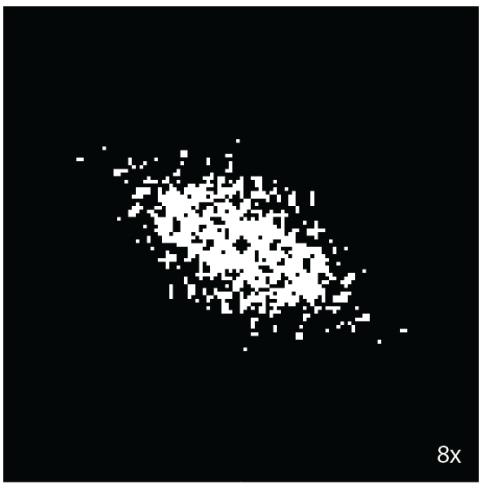

FIG. 2. Fourier transform examples. (a) Points arranged in a spatially periodic array. Blue and red bars highlight some periods. (b) Fourier spectrum of panel (a) with Gaussian blur of standard deviation 0.6. (c) Same as panel (b) after selecting a proportion $p$ of the image pixels which are the brightest, with $p=7 \times 10^{-6}$. The two principal directions are visible. (d) Anisotropic myosin distribution in a chicken embryo during morphogenesis, courtesy of C. J. Weijer. (e) Fourier spectrum of panel (d) with Gaussian blur of standard deviation 0.6. (f) Same as panel (e) after selecting a proportion $p$ of the image pixels which are the brightest, with $p=10^{-3}$. In panels (b), (c), (e), (f), " $8 \times$ " signifies that the spectra are zoomed eight times.

pattern (such as fibers) is a single broad peak centered on zero frequency [Figs. 2(d)-2(f)]; the distribution width reveals the range of length scales present in the image, and the distribution anisotropy reveals the fiber anisotropy.

An epithelial tissue pattern [Fig. 3(a)] is aperiodic and usually lies between these extremes. The analysis is performed on overlapping subimages [Figs. 3(a) and 3(b)]. The window size is chosen as a trade-off between the signal-to-noise ratio and the spatial resolution required to answer the question under consideration. The Fourier transform uses a periodic image, but in practice opposite borders of a window are different. This causes artifacts-such as a cross on the FT spectra-that are avoided by a periodic plus smooth image decomposition [26]. The FT phase provides information on the cell junction positions within each subimage, which is not of interest here. We keep only the FT amplitude. In Fourier space, we represent the FT power spectrum density, with the zero frequency at the center [Fig. 3(c)]. We perform a time average over successive images; their number is chosen as a trade-off between the signal-to-noise ratio and time resolution required to answer the question under consideration. When the experiment is repeated, we average the FT spectrum of the different available samples ("ensemble average"); as opposed to space and time averages, ensemble averaging has only advantages in terms of signal-to-noise ratio.
The resulting power spectrum density is smoothed with a Gaussian blur [Fig. 3(d)]. Low spatial frequencies, corresponding to length scales much larger than a cell size, are removed [Fig. 3(e)]. The FT anisotropy reflects the pattern anisotropy; the FT itself is a blurred ring [Figs. 3(c)-3(e)], more or less resolved depending on the initial image quality and cell area variance. This enables the two following possibilities for the analysis of cell anisotropy.

The first method, called the "FT ellipse ring fit," also yields access to cell size. It applies to a cellular pattern with disordered cell-cell junction orientation, a small variance in area and a good image contrast, then the FT is a well-resolved elliptic ring which can be fit by an ellipse [Fig. 3(f)]. Its axis sizes in Fourier space are $\left(\mu_{\text {maj }}, \mu_{\text {min }}\right)$. They yield, back in real space, the ellipse axis sizes which describe the average cell properties within the subimage [Fig. $3(\mathrm{~g})]: L_{\text {maj }}=\frac{2 m}{\mu_{\text {min }}}$, $L_{\text {min }}=\frac{2 m}{\mu_{\text {maj }}}$; here $m$ is the size of the FT image in pixels. To ensure the link with the real absolute size, $L_{\text {maj }}$ and $L_{\min }$ have to be multiplied by the pixel size. The angle between the $x$ axis and major axis is $\theta$ in real space and $\theta+\pi / 2$ in Fourier space.

The second method, called the "FT inertia matrix," is more general because it applies even if the FT ellipse ring is illresolved, as in Fig. 2(f). From Fig. 3(e), we keep a percentile 

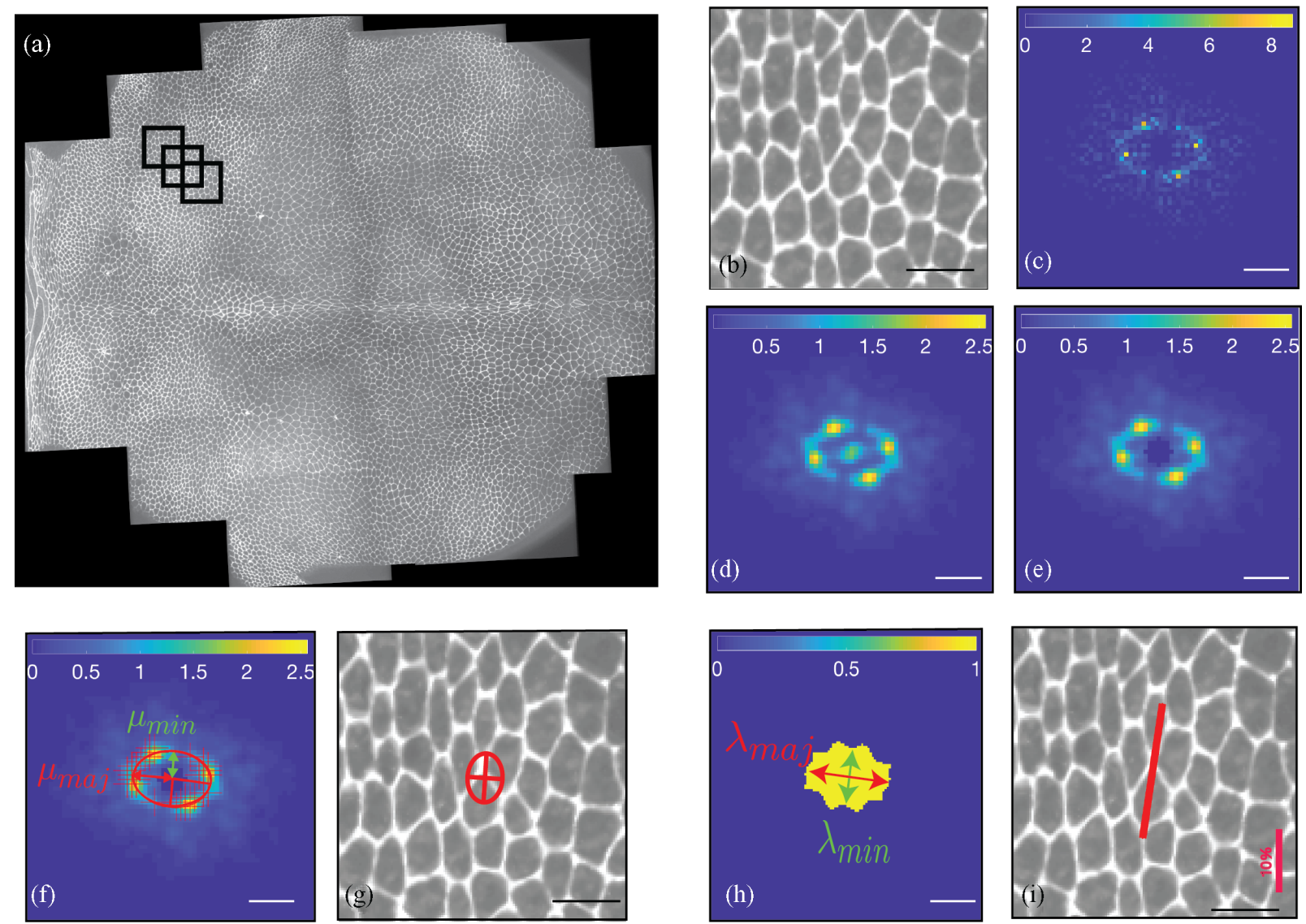

FIG. 3. Fourier-transform-based pipeline. (a) Whole segmented image in original gray levels, same data as Figs. 1(a) and 1(b) [10]. Boxes are examples of subwindows, showing their overlap. (b) Image in a subwindow, (c) its power spectrum density, (d) same after a Gaussian blur of standard deviation 1.2, (e) same after suppression of low frequencies (dark zone in the center). (f) An ellipse is fit to the ring; its axis orientations and lengths $\left(\mu_{\mathrm{min}}, \mu_{\mathrm{maj}}\right)$ in Fourier space define an ellipse with the same axis orientations and inverse axis lengths in real space. $(\mathrm{g})$ In real space, the ellipse size corresponds to the average cell size. (h) Thresholding the image, filling the ellipse, and measuring its FT inertia matrix determines the pattern anisotropy, quantified by the ratio of ellipse axes $\lambda_{\min } / \lambda_{\text {maj }}$ in the Fourier space, and the direction $\theta$ of the ellipse axes. Returning to the real space (i), the pattern anisotropy is determined: its magnitude is represented by the bar size, and its direction is the direction of the bar. For panels (c)-(f) and (h), the white scale bar is $0.1 \mu \mathrm{m}^{-1}$, for panels (b), (g), and (i), the black scale bar is $10 \mu \mathrm{m}$, for panel (i), the red scale bar is $10 \%$ of elongation.

$p$ of the image pixels which are the brightest (hereafter called "proportion" for short), to threshold the spectrum. A morphological closing is then performed to remove the gaps between points [Fig. 3(h)]. The resulting binarized pattern defines a filled ellipse with a correct aspect ratio. Then, the inertia matrix (see Appendix A) of the filled ellipse is computed and yields a major $\left(\lambda_{\text {maj }}\right)$ and minor axis $\left(\lambda_{\min }\right)$. Returning to the real space, the ellipse axes $L_{\text {maj }}=\frac{2 m}{\lambda_{\min }}$ and $L_{\text {min }}=\frac{2 m}{\lambda_{\text {maj }}}$ define anisotropy. Again, the angles of eigenvectors, $\theta$ and $\theta+\pi / 2$, are the same in Fourier and real spaces. Note that here $L_{\text {min }}$ and $L_{\text {maj }}$ have no meaning in terms of absolute cell size, because they are entirely dependent on the proportion parameter. However, they reflect the pattern anisotropy, as we will now discuss.

There are several families of acceptable definitions of internal strain [27]. Among them, one contains an infinity of acceptable definitions that are functions of $L_{\text {maj }}, L_{\min }$ [28]. We choose the "true" strain that was first introduced in the engineering field to describe large strains [29]. By using the true strain formalism the anisotropic part of the cell strain is defined as a matrix with the same eigenvectors as the FT and with eigenvalues $\pm \frac{1}{2} \ln \frac{L_{\text {maj }}}{L_{\text {min }}}$ (see Appendix A). The absolute value of this amplitude (or its linearized approximations, if the strain is small; see Appendix A) is used as a measure of anisotropy, which we represent as a bar in the direction $\theta$ [Fig. 3(i)]. The results are sensitive to the proportion $p$ of pixels kept for thresholding. However, a reasonable range of values of $p$ allows for a robust determination of anisotropies (see Appendix B).

Altogether, the parameters which must be adjusted for both methods are window size and overlap, time average, Gaussian blur size, low cutoff for spatial frequencies; in addition, for FT ellipse ring fit: number of fit points; and for FT inertia matrix: proportion for thresholding, dilation-erosion size. The code is available on Github [30]. It is user friendly and optimized to reduce the time it takes to manually adjust the parameters, typically five minutes at the beginning and one minute when the user is trained. Once these parameters are adjusted for a first image, they can be reused for all similar images of the same series. 
(a)

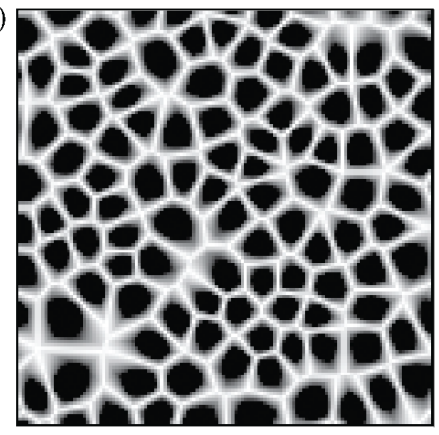

(b)

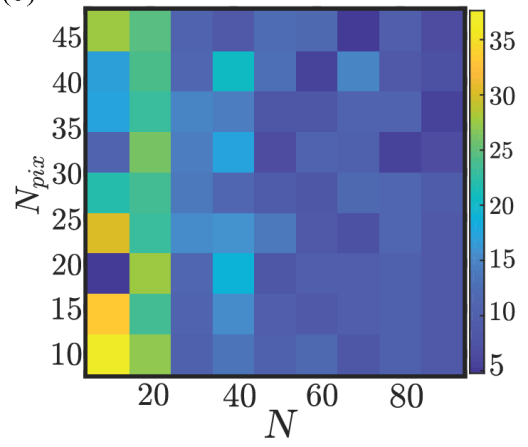

(c)

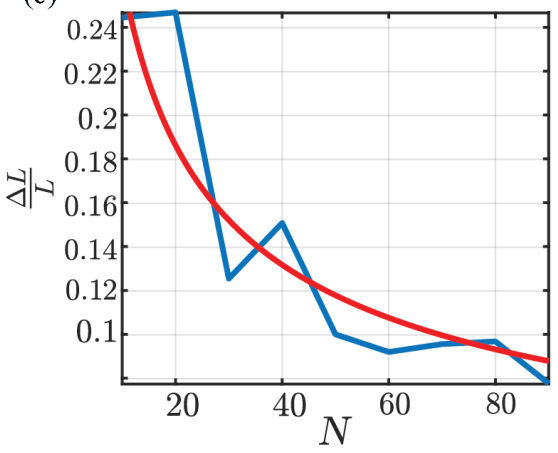

FIG. 4. Precision on cell size determination. (a) Example of an artificial cellular pattern. Window size 128 pixels, at least 8 pixels between seeds, packing fraction $0.5, N=80$ cells, $N_{\text {pix }}=158$ pixels per cell in average. (b) Relative error on the cell size: $\frac{\Delta L}{L}$ in percentage, where $L$ is the average radius of the cells in the image vs number $N$ of cells in the image and average number $N_{\text {pix }}$ of pixels per cells. Data from 1260 images (126 series of 10 repeats). Each square is an average with a minimum of 10 images. (c) Blue is relative error vs $N$ for all images, i.e., averaged on $N_{\text {pix }}$. Red is best fit by a $N^{-1 / 2}$ law, with a prefactor of 0.83 .

\section{RESULTS}

\section{A. Precision on cell size determination}

To test the precision on cell size determination, we first run the pipeline on a set of artificial cellular patterns.

Each image is created on a square of side $L_{\text {pix }}$ pixels by sequentially placing $N$ seeds at random points, with a minimum distance between them. Their Voronoi diagram is created, and the cell-cell junctions are thickened to reach a prescribed packing fraction [Fig. 4(a)]. We measure the number of pixels per cell and the average cell size on the pattern. We then apply our pipeline and compute its error in cell size determination. This test is repeated on a series of ten images with the same parameters (minimum distance between seeds, and packing fraction). Then the parameters are varied to generate a set of 126 different series.

Since we measure the cell size $L$ from a peak in the FT, we expect the peak position in Fourier space to be around $1 / L$. The precision in peak position determination is of the order of one pixel in Fourier space, i.e., $\frac{1}{L \sqrt{N}}$ back in real space. The relative error on $L$ is thus of order $N^{-1 / 2}$, independently of the number of pixels per cell, $N_{\text {pix }}$. This is consistent with the results of our tests, where the value of $N_{\text {pix }}$ has no effect as soon as it is larger than 20 [Fig. 4(b)] and the value of $\Delta L / L$ is of the order of $0.83 N^{-1 / 2}$ [Figs. 4(b) and 4(c)]. Note that it would be possible to increase the resolution by padding the image - adding zeros around the picture [31]. This simple process allows the pixel size in Fourier space to be changed, and thus gives access to different ranges of frequency: it can improve the Fourier transform resolution and allow a subpixel accuracy to be reached back in real space. It is not used in the present article nor in the online code.

\section{B. Validation of cell size and anisotropy determination}

To validate the cell size and anisotropy determination methods, we run the pipeline on an image [Fig. 3(a)] whose segmentation [Fig. 1(a)] quality makes it a gold standard [10]. The FT calculation has been performed in Matlab on a OSX with an Intel Core $i 7$ processor at $2.2 \mathrm{GHz}$ clock frequency. It takes about 60 seconds for the computation of the anisotropy part alone with the inertia matrix method, about 40 seconds for the computation of the size alone with the ellipse ring fit method.

The cell anisotropy measurements using FT inertia matrix methods correlate with the segmentation measurements, qualitatively [Figs. 5(a) and 5(b)] and quantitatively [Figs. 5(c) and 5(d)] in amplitude and orientation. The cell size and anisotropy (amplitude and orientation) measurements using the FT ellipse ring fit correlate well with the segmentation measurements, qualitatively [Figs. 5(e) and 5(f)] and quantitatively [Figs. 5(c) and 5(g)]. As expected, the anisotropy orientation measurement is better when the anisotropy amplitude is larger; at small anisotropies the FT ellipse ring fit performs better than the FT inertia matrix [Fig. 5(c)].

\section{Measurements on a large dataset}

We now test the FT analysis on a case where the cell number is particularly large. Data come from chicken morphogenesis, or more precisely from a study of cell flows during primitive streak formation, estimating the relative contributions of cell shape changes and cell neighbor rearrangements [32]. Each image contains hundreds of thousands of cells [Fig. 6(a)]. Altogether, taking into account wild-type and mutant conditions, hundreds of movies have been acquired, each with hundreds of images, resulting in several billion cells. The image quality and contrast are good enough for segmentation, but the cell number is too large and segmentation has been performed only on a subset of images.

Most boxes display clearly isotropic cell shapes [Fig. 6(b)], as quantitatively confirmed by their anisotropy [Fig. 6(c)]. However, several boxes contain cells clearly displaying a shape anisotropy [Fig. 6(d)], as again quantitatively confirmed [Fig. 6(e)]. These anisotropic cells are all located in a band, perpendicular to the anteroposterior (AP) axis, the so-called sickle region undergoing an extension. The measurement which is sought here is the position and width of this extension region. This does not require segmentation, and FT analysis seems appropriate. 


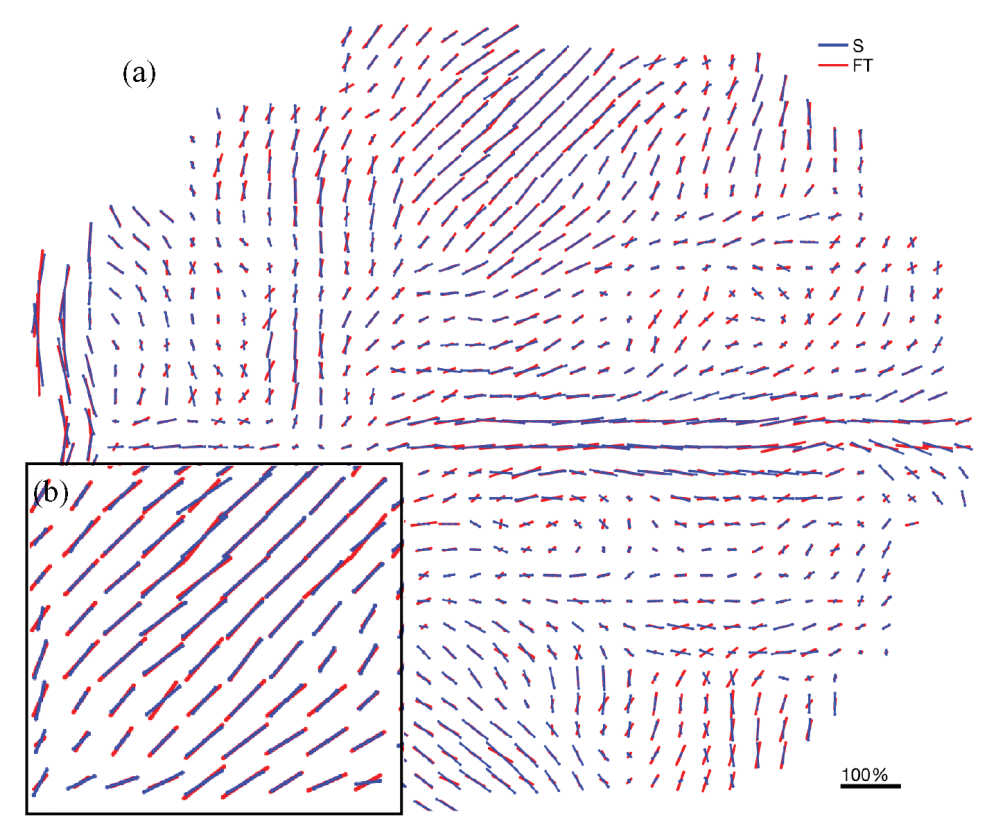

(e)

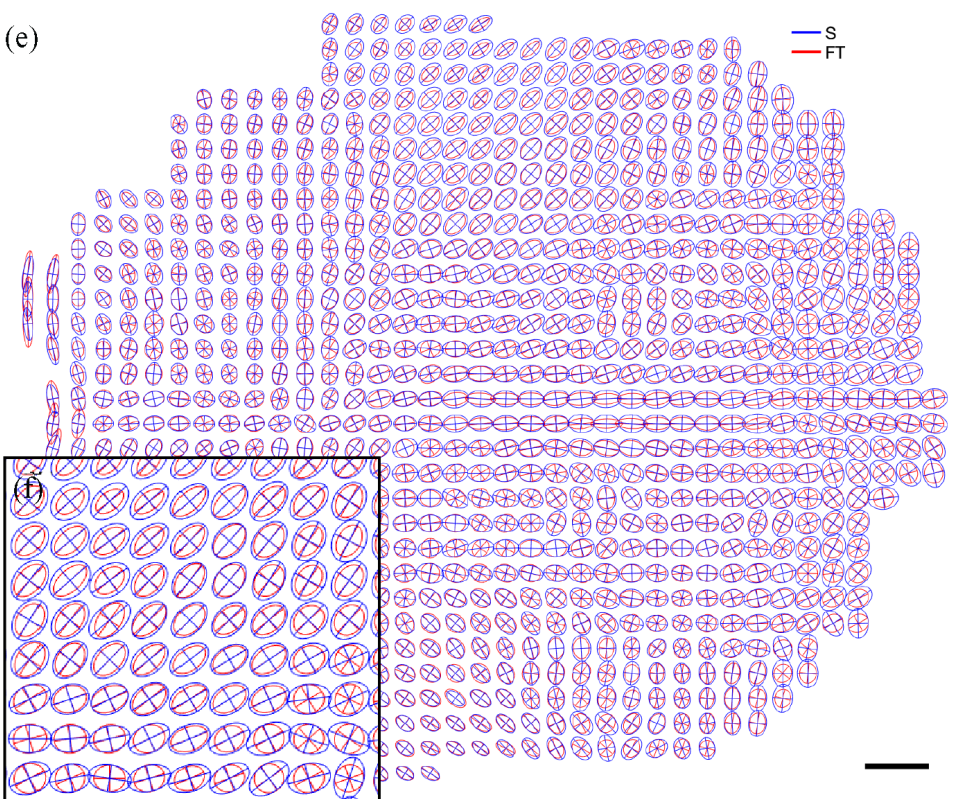

(c)

(d)
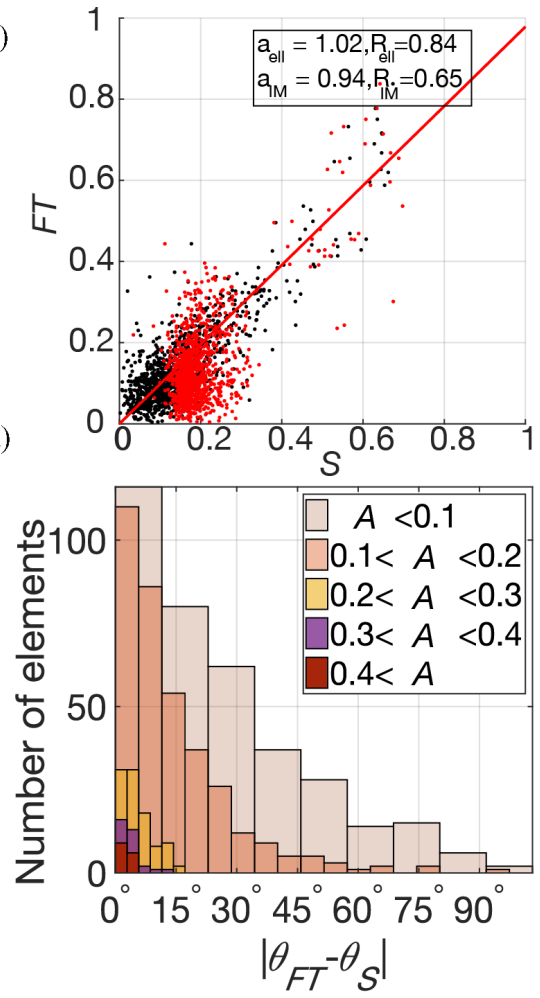

(g)

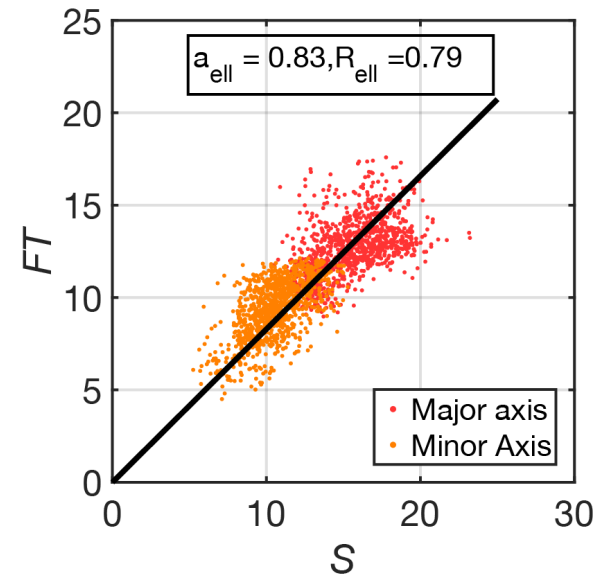

FIG. 5. Test of cell anisotropy and size measurements. (a) Map of cell anisotropies on the image in Fig. 1(a). There are 1221 boxes of $128 \times 128$ pixels with a $50 \%$ overlap. Blue bars are from segmentation; data of Fig. 1(a). Red bars are from FT analysis computed with the inertia matrix. (b) Close-up of a region of panel (a). (c) Anisotropy measurements using FT ellipse fit ring (black) and FT inertia matrix (red) vs measurements using segmentation. Each point corresponds to a box. The slopes of the fit are $1.02(R=0.84)$ for FT ellipse fit ring and $0.94(R=0.65)$ for FT inertia matrix. (d) Histogram of the difference between angles from FT with the inertia matrix method and from segmentation. The color codes for the anisotropy amplitude $A$. (e) Map of cell ellipses, representing cell sizes and anisotropies. Blue shows results from segmentation; data of Fig. 1(a). Red shows results from FT ellipse ring fit method, plotted as ellipses in real space. Scale bar is $50 \mu \mathrm{m}$. (f) Close-up of a region of panel (e). (g) Major axis (red) and minor axis (orange) measurements using FT ellipse fit vs measurements using segmentation. Each point corresponds to a box; the slope of the fit is $0.83(R=0.79)$.

Taking advantage of the expected band structure, we average the FT spectrum [Fig. 6(f)] over boxes in a rectangle perpendicular to the AP axis, strongly improving the signalto-noise ratio [Fig. 6(g)]: the ellipse ring becomes visible. We have the choice between both methods and choose here the
FT inertia matrix, more robust than the FT ellipse ring fit to variations in image quality and contrast and sufficient to answer the questions under consideration. By thresholding, calculating the inertia matrix, and diagonalizing it, we measure the anisotropy of the average FT (not the average of FT 


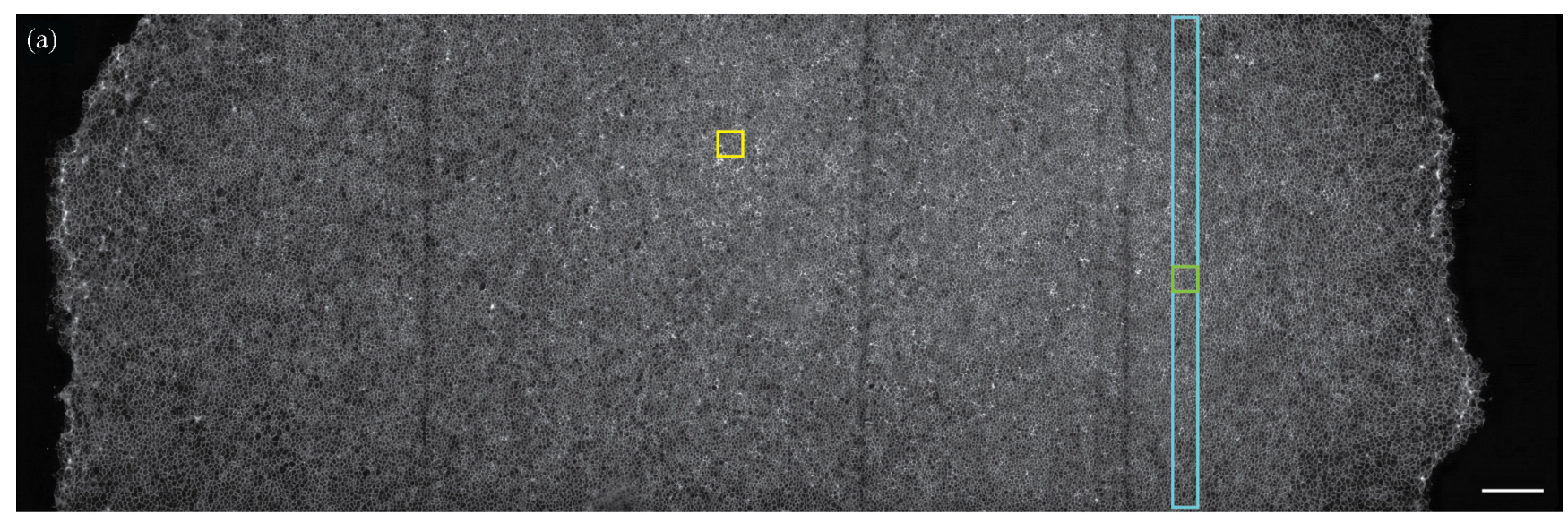

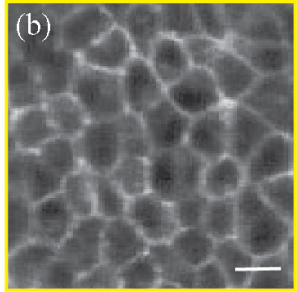

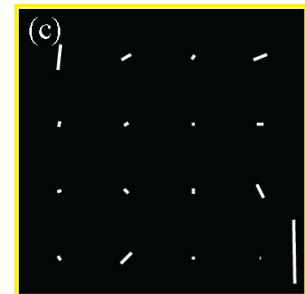

(f)

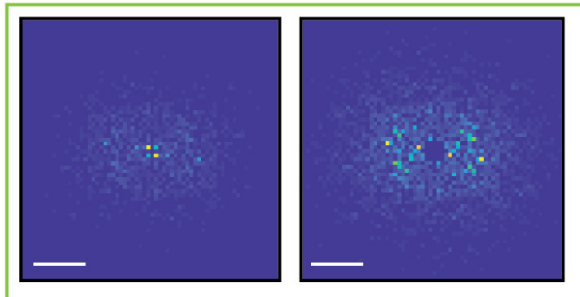

(h)

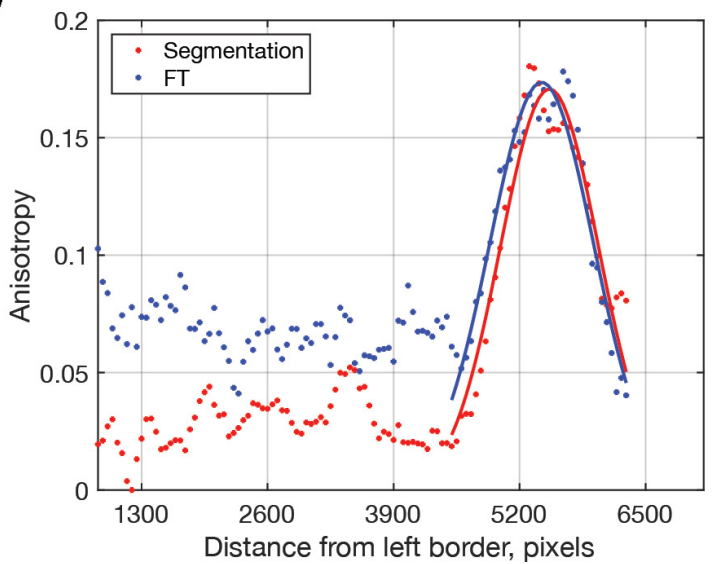

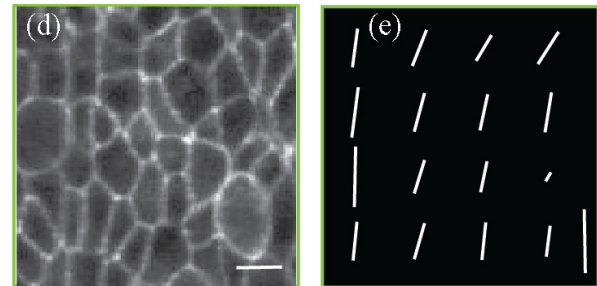

(g)

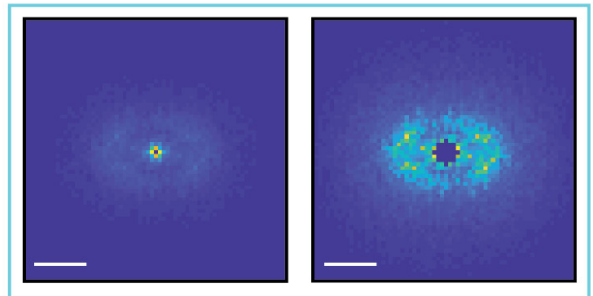

(i)

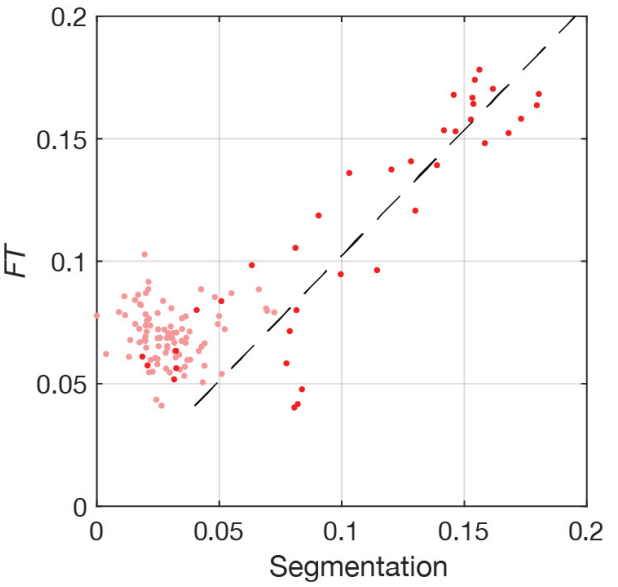

FIG. 6. Large cell number anisotropy measurements. (a) Light sheet microscopy image of a chicken embryo at cellular resolution [32]. Scale bar is $200 \mu \mathrm{m}$. The field of view contains on the order of $10^{5}$ cells. The anteroposterior (AP) axis is horizontal, with the anterior on the left, posterior on the right. The two framed squares are $200 \times 200$ pixels ( $\sim 100$ cells). (b), (c) Close-up of yellow framed square in panel (a), and corresponding anisotropy measured by FT inertia matrix at several positions. (d), (e) Same as panels (b) and (c) for green framed square in panel (a), in the sickle region. For panels (b) and (d), the scale bar is $20 \mu \mathrm{m}$; for panels (c) and (e), the scale bar is $10 \%$ elongation. (f) Left: raw Fourier spectrum of the green framed square in $A$. Right: Same after removing small spatial frequencies and adapting the color scale. White scale bar is $10^{-1} \mu \mathrm{m}^{-1}$. (g) Same as panel (f), averaged over the whole blue framed rectangle in panel (a), perpendicular to the AP axis. (h) Anisotropy vs position along the AP axis. Averages are performed perpendicularly to the AP axis, as in panel (g), and the anisotropy computed after averaging. Red shows measurements using segmentation; blue shows measurements using FT inertia matrix; lines are Gaussian fits to the region between 4500 and 6300 pixels from left border. (i) Measurements using FT inertia matrix vs measurements using segmentation. Dashed line is linear fit the same data as in (h) (in red), slope $1.02(R=0.89)$. 

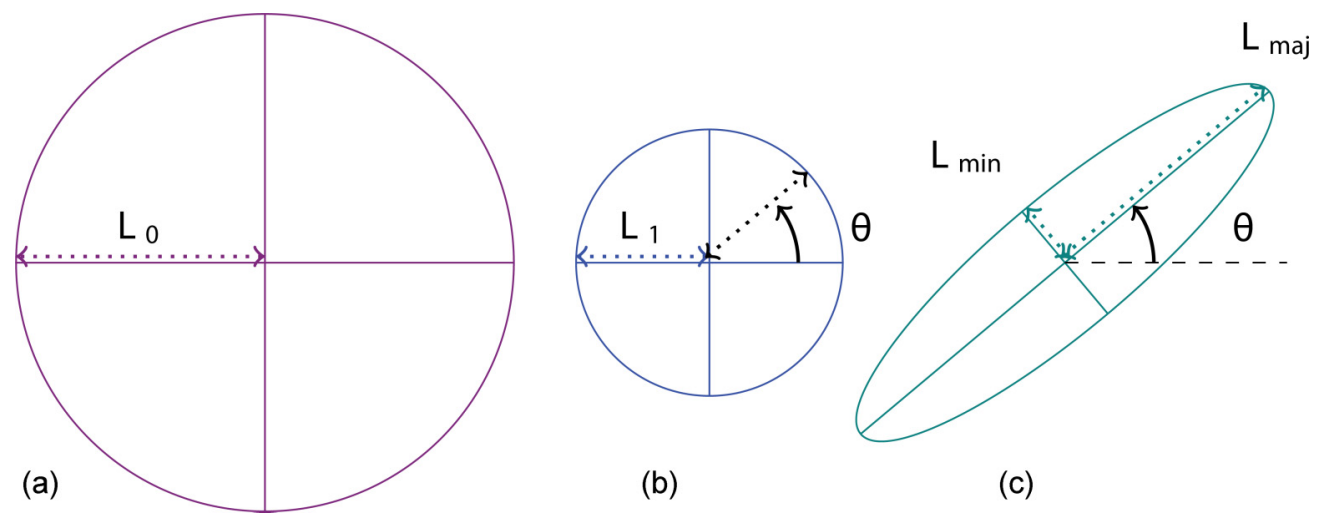

FIG. 7. Strain: isotropic and anisotropic contributions. Under a purely isotropic deformation, or growth (positive or negative), (a) a disk of radius $L_{0}$ transforms into (b) a disk of radius $L_{1}$. Under a purely anisotropic deformation, or convergence-extension, (b) a disk of radius $L_{1}$ transforms into (c) an ellipse of major axis $L_{\text {maj }}$, in direction $\theta$, and minor axis $L_{\min }$, in the perpendicular direction, with area conservation expressed by $L_{\text {maj }} L_{\min }=L_{1}^{2}$.

anisotropy). The calculation has been performed on an OSX with an Intel Core i 7 processor at $2.2 \mathrm{GHz}$ clock frequency. It takes three minutes in Matlab to compute the anisotropy part alone with the inertia matrix method.

FT provides the whole profile of anisotropy along the $x$ axis; it clearly reveals the existence and estimates the position and width of the band, in good qualitative agreement with the segmentation [Fig. 6(h)]. Quantitatively, for boxes with anisotropy at least equal to 0.08 , the agreement between FT and segmentation results is very good [Figs. 6(h) and 6(i)]: for data obtained with segmentation, the Gaussian fit peak position is at $5500 \pm 11 \mu \mathrm{m}$ and its standard deviation is $\sigma=358 \pm 23 \mu \mathrm{m}$, while for the FT inertia matrix data, the peak position is at $5450 \pm 10 \mu \mathrm{m}$ and $\sigma=377 \pm 20 \mu \mathrm{m}$.

\section{CONCLUSION}

In summary, we present a Fourier-transform-based analysis pipeline to measure the coarse-grained field of pattern anisotropies. It applies in particular to fixed or live, fluorescent and phase contrast images of epithelial tissues, in which it characterizes the coarse-grained cell anisotropy. One variant, the "inertia matrix" method, applies even when the image quality is low. The other variant, the "ellipse ring" method, yields also the coarse-grained cell size, and performs better at small anisotropies.

After a user-friendly manual tuning of a few parameters, it can automatically handle tens of thousands of cells per minute. We successfully validated it against segmentationbased measurements. It is robust against defects in image contrast, heterogeneities in cell sizes and orientations, choice of parameters. It is adequate to improve the signal-to-noise ratio using space, time and/or ensemble averages; the latter are averages over samples and do not deteriorate the time or space resolution.

Possible applications include ordered tissues, real-time analysis of live movies, in vitro tissues, nonliving cellular materials such as liquid foams; extensions of applications could include $x y z$ stacks of tridimensional tissues. Moreover, assuming that in the rest state the coarse-grained cell shape is isotropic, the pipeline can also yield access to the cell strain deviator, a mechanical measure important to characterize a tissue, as shown in a companion paper [33].

\section{ACKNOWLEDGMENTS}

We warmly thank C. J. Weijer for providing chicken embryo images and for critical reading of the paper. We also thank F. Bosveld, S. Rigaud, and Y. Bellaïche for their role in acquiring Drosophila pupa data.

\section{APPENDIX A: MATRICES USED IN THE TEXT}

We introduce here three types of $2 \times 2$ matrices, also called rank-2 tensors: the inertia matrix, the FT inertia matrix, and the cell strain.

The inertia matrix of a binarized pattern is defined by

$$
I=\left(\begin{array}{ll}
\langle x x\rangle & \langle x y\rangle \\
\langle x y\rangle & \langle y y\rangle
\end{array}\right) .
$$

Here $x$ and $y$ are coordinates with the origin at the pattern barycenter, $\langle x\rangle=\langle y\rangle=0$. The brackets indicate an average over the coordinates within the pattern (if the pattern were in gray levels instead of being binarized, the average would be weighted by the gray levels). The four terms which appear in $I$ are the coordinate covariances.

The Fourier transform inertia matrix has the same definition, Eq. (A1), but it operates in the Fourier space. Here $x$ and $y$ are coordinates in the space of spatial frequencies, again with the origin at the pattern barycenter, $\langle x\rangle=\langle y\rangle=0$.

The cell strain has isotropic and anisotropic contributions (Fig. 7):

$$
\varepsilon_{c}=\frac{1}{2} \operatorname{Tr}\left(\varepsilon_{c}\right) \mathcal{I}_{2}+\varepsilon_{c}^{\mathrm{dev}},
$$

where $\mathcal{I}_{2}$ is the identity matrix in two dimensions and $\operatorname{Tr}$ is the trace.

Consider a circle of radius $\ell$ (Fig. 7) and apply a small variation of its length $d \ell$. Its relative extension is $d \ell / \ell=d(\ln \ell)$. Integrating this infinitesimal extension between the initial and final states yields the expression for strain [34], which for the isotropic part is written [Figs. 7(a) 


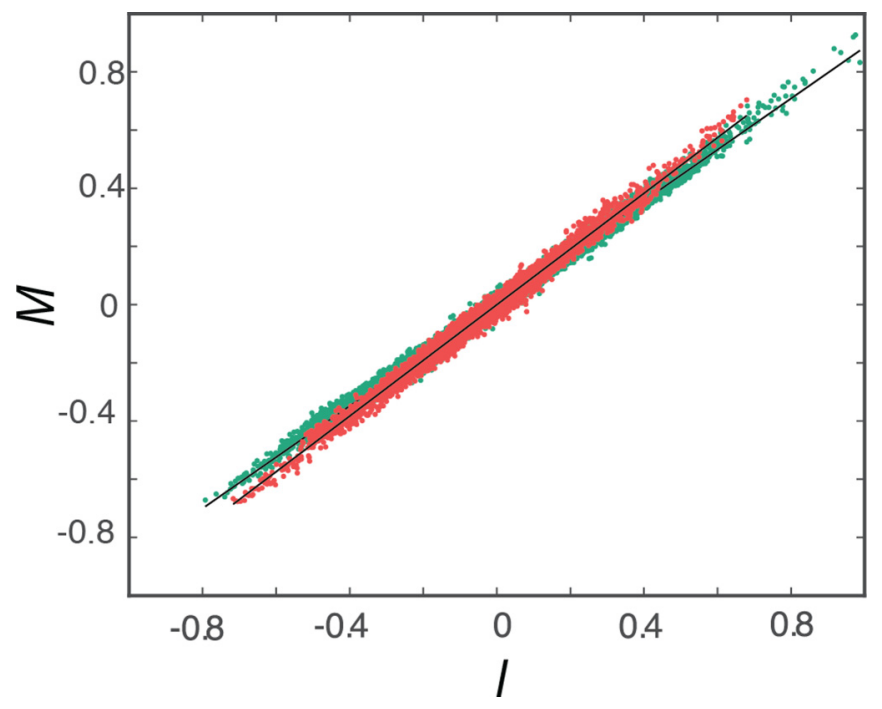

FIG. 8. Texture tensor versus inertia matrix. Data presented here are from the same dataset as Fig. 1. Diagonal component (green) and off-diagonal component (red) of the texture tensor $M$ as defined in Ref. [18] versus the corresponding anisotropic (diagonal and offdiagonal) component of the inertia matrix $I$, representing cell shape anisotropy. Slopes are 0.88 and 0.96 , correlations coefficients are 0.99 and 0.98 , respectively. Tensors built with data from Ref. [10], here plotted after adimensionalization by the isotropic part of the respective tensor. To compute each of the 14112 points, tensors of individual cells are computed and then averaged in Eulerian grids of $40 \times 40 \mu \mathrm{m}^{2}$ with $50 \%$ overlap. Then a sliding average is performed on $2 \mathrm{~h}$ ( 24 frames) of time with a one hour overlap. Boxes at the pattern boundary which are filled at less than $30 \%$ by cells are excluded from the fit.

and 7(b)]

$$
\frac{1}{2} \operatorname{Tr}\left(\varepsilon_{c}\right) \mathcal{I}_{2}=\frac{\ln \left(L_{1} / L_{0}\right)}{2}\left(\begin{array}{ll}
1 & 0 \\
0 & 1
\end{array}\right),
$$

and for the anisotropic part, after diagonalization along axes of directions $\theta$ and $\theta+\pi / 2$ [Figs. 7(b) and 7(c)]:

$$
\begin{aligned}
\varepsilon_{c}^{\operatorname{dev}} & =\left(\begin{array}{cc}
\ln \left(\frac{L_{\text {maj }}}{L_{1}}\right) & 0 \\
0 & \ln \left(\frac{L_{\text {min }}}{L_{1}}\right)
\end{array}\right) \\
& =\left(\begin{array}{cc}
\frac{\ln \left(L_{\text {maj }} / L_{\text {min }}\right)}{2} & 0 \\
0 & -\frac{\ln \left(L_{\text {maj }} / L_{\text {min }}\right)}{2}
\end{array}\right),
\end{aligned}
$$

where $L_{1}=\sqrt{L_{\text {maj }} L_{\text {min }}}$. The cell strain deviator amplitude is $\frac{1}{2} \ln \frac{L_{\text {maj }}}{L_{\text {min }}}$, and the cell strain deviator orientation is $\theta$. The cell strain deviator can be inferred from the pattern anisotropy, without requiring any information about the current cell size $L_{1}$ or its rest state value $L_{0}$; the above derivation only assumes that the rest state is isotropic. Note that this definition of the strain is called the "true" strain, or Hencky strain [29]. When

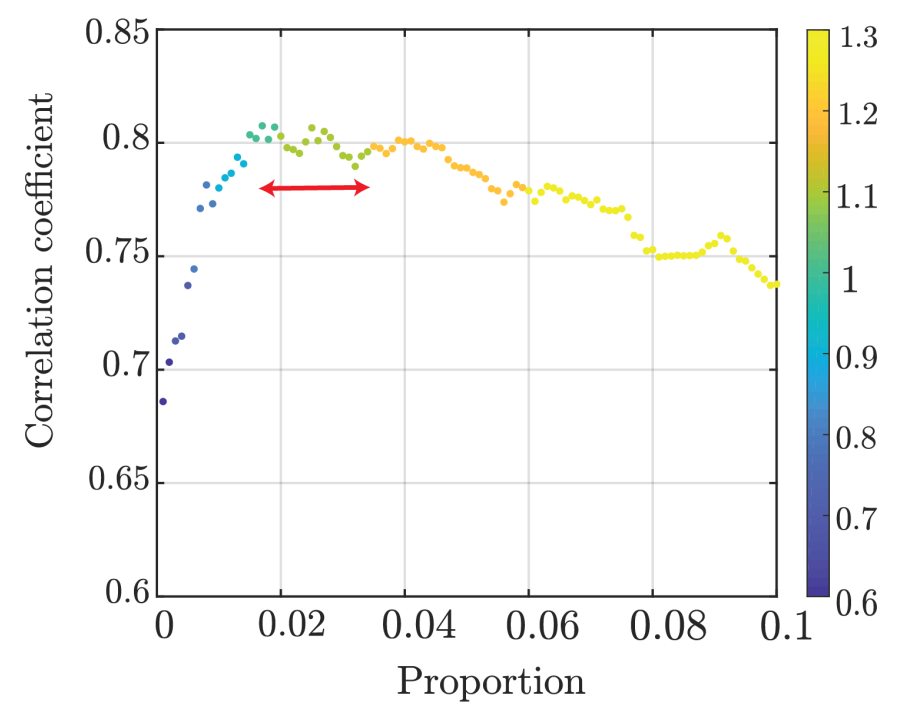

FIG. 9. Robustness of the inertia matrix method quality versus choice of parameter: correlation coefficient versus "proportion" parameter (see text), for the Drosophila pupa data set. The correlation slope is color coded. The method quality is optimal when the correlation coefficient is high and the slope close to 1 . The red arrow represents the parameter range which can reasonably be used for this data set.

the cell strain deviator amplitude is much smaller than unity, one can alternatively use any approximation equivalent at first order, such as $\frac{1}{2}\left(\frac{L_{\mathrm{maj}}}{L_{\mathrm{min}}}-1\right)$, for instance when using the linear approximation to the true strain, or $\frac{1}{4}\left(\frac{L_{\text {maj }}^{2}}{L_{\text {min }}^{2}}-1\right)$, when using quadratic tensors attached to the matter: inertia matrix, defined in Eq. (A1), or texture tensor, defined in Ref. [18]. We have also checked that the inertia matrix and the texture tensor statistically yield equivalent information (Fig. 8).

\section{APPENDIX B: ROBUSTNESS OF INERTIA MATRIX METHOD}

Figure 9 investigates the robustness of the inertia matrix method quality versus the choice of the main parameter, the manually selected proportion $p$ of bright pixels used when thresholding the Fourier transform [Fig. 3(h)]. Using the Drosophila pupa dataset, for each value of the proportion we compare the inertia matrix method results with the segmentation analysis considered as a gold standard, by performing a linear regression on data with anisotropy larger than 0.08 . The method quality is considered as optimal when the linear regression has a slope close to 1 and its correlation coefficient is high. We find an optimum for a proportion around $2 \times 10^{-2}$ and a large parameter range around this value where the method quality is robust (red arrow).
[1] C.-P. Heisenberg and Y. Bellaïche, Cell 153, 948 (2013).

[2] J. A. Zallen and R. Zallen, J. Phys.: Condens. Matter 16, 5073 (2004).
[3] A.-K. Classen, K. I. Anderson, E. Marois, and S. Eaton, Dev. Cell 9, 805 (2005).

[4] T. Hayashi and R. W. Carthew, Nature 431, 647 (2004). 
[5] J. Käfer, T. Hayashi, A. F. M. Marée, R. W. Carthew, and F. Graner, Proc. Natl. Acad. Sci. U.S.A. 104, 18549 (2007).

[6] S. Hilgenfeldt, S. Erisken, and R. W. Carthew, Proc. Natl. Acad. Sci. U.S.A. 105, 907 (2008).

[7] R. Farhadifar, J.-C. Röper, B. Aigouy, S. Eaton, and F. Jülicher, Curr. Biol. 17, 2095 (2007).

[8] M. Rauzi, P. Verant, T. Lecuit, and P.-F. Lenne, Nat. Cell Biol. 10, 1401 (2008).

[9] G. B. Blanchard, A. J. Kabla, N. L. Schultz, L. C. Butler, B. Sanson, N. Gorfinkiel, L. Mahadevan, and R. J. Adams, Nat. Methods 6, 458 (2009).

[10] B. Guirao, S. U. Rigaud, F. Bosveld, A. Bailles, J. López-Gay, S. Ishihara, K. Sugimura, F. Graner, and Y. Bellaïche, eLife 4, e08519 (2015).

[11] R. Etournay, M. Merkel, M. Popović, H. Brandl, N. A. Dye, B. Aigouy, G. Salbreux, S. Eaton, and F. Jülicher, eLife 5, e14334 (2016).

[12] E. Faure, T. Savy, B. Rizzi, C. Melani, O. Stašová, D. Fabrèges, R. Špir, M. Hammons, R. Č́nderlík, G. Recher, B. Lombardot, L. Duloquin, I. Colin, J. Kollár, S. Desnoulez, P. Affaticati, B. Maury, A. Boyreau, J.-Y. Nief, P. Calvat, P. Vernier, M. Frain, G. Lutfalla, Y. Kergosien, P. Suret, M. Remešíková, R. Doursat, A. Sarti, K. Mikula, N. Peyriéras, and P. Bourgine, Nat. Commun. 7, 8674 (2016).

[13] K. Sherrard, F. Robin, P. Lemaire, and E. Munro, Curr. Biol. 20, 1499 (2010).

[14] M.-D.-C. Diaz de la Loza, R. P. Ray, P. S. Ganguly, S. Alt, J. R. Davis, A. Hoppe, N. Tapon, G. Salbreux, and B. J. Thompson, Dev. Cell 46, 23 (2018).

[15] D. Heller, A. Hoppe, S. Restrepo, L. Gatti, A. L. Tournier, N. Tapon, K. Basler, and Y. Mao, Dev. Cell 36, 103 (2016).

[16] S. Ishihara, P. Marcq, and K. Sugimura, Phys. Rev. E 96, 022418 (2017).

[17] E. Latorre, S. Kale, L. Casares, M. Gómez-González, M. Uroz, L. Valon, R. V. Nair, E. Garreta, N. Montserrat, A. del
Campo, B. Ladoux, M. Arroyo, and X. Trepat, Nature 563, 203 (2018).

[18] F. Graner, B. Dollet, C. Raufaste, and P. Marmottant, Eur. Phys. J. E 25, 349 (2008).

[19] R. O. Duda and P. E. Hart, Commun. ACM 15, 11 (1972).

[20] S. J. Streichan, M. F. Lefebvre, N. Noll, E. F. Wieschaus, and B. I. Shraiman, eLife 7, e27454 (2018).

[21] R. Lehoucq, J. Weiss, B. Dubrulle, A. Amon, A. Le Bouil, J. Crassous, D. Amitrano, and F. Graner, Front. Phys. 2, 84 (2015).

[22] A. Boudaoud, A. Burian, D. Borowska-Wykrȩt, M. Uyttewaal, R. Wrzalik, D. Kwiatkowska, and O. Hamant, Nature Protocols 9, 457 (2014).

[23] F. Bosveld, I. Bonnet, B. Guirao, S. Tlili, Z. Wang, A. Petitalot, R. Marchand, P.-L. Bardet, P. Marcq, F. Graner, and Y. Bellaïche, Science 336, 724 (2012).

[24] A. Bruns, J. Neurosci. Methods 137, 321 (2004).

[25] A. Bruns, J. Neurosci. Methods 143, 237 (2005).

[26] L. Moisan, J. Math. Imaging Vis. 39, 161 (2011).

[27] K. Bagi, Int. J. Solids Struct. 43, 3166 (2006).

[28] K. Farahani and R. Naghdabadi, Int. J. Solids Struct. 37, 5247 (2000).

[29] H. Hencky, J. Rheol. 2, 169 (1931).

[30] M. Durande, Github Codes for Fast Determination of CoarseGrained Cell Anisotropy and Size in Epithelial Tissue Images using Fourier Transform, https://github.com/mdurande/ coarse-grained-anisotropy-and-size-using-FFT (2019).

[31] S. Hilbert, FFT Zero Padding, http://www.bitweenie.com/ listings/fft-zero-padding/? (2013).

[32] E. Rozbicki, M. Chuai, A. I. Karjalainen, F. Song, H. M. Sang, R. Martin, H.-J. Knölker, M. P. MacDonald, and C. J. Weijer, Nat. Cell Biol. 17, 397 (2015).

[33] S. Tlili, C. Gay, B. Ladoux, F. Graner, and H. Delanoë-Ayari, arXiv:1811.05001.

[34] R. Tanner and E. Tanner, Rheol. Acta 42, 93 (2003). 